\title{
Numerical Modeling of the Transition between Free Surface and Pressurized Flow in Storm Sewers
}

Jose G. Vasconcelos and Steven J. Wright

Stormwater drainage systems are typically composed of a network of closed conduits designed to operate under normal conditions in an open-channel, freesurface flow regime. Nevertheless, during intense rain events, the conduits may fill completely, undergoing a transition to a closed-pipe, pressurized flow. For sufficiently rapid filling, this transition will occur through a moving interface that advances into the free-surface portions of the system. In particular, if the system geometry restricts the escape of air ahead of the advancing front, the air pressurization will induce a motion in the underlying water.

The complexity of the system geometries and the flow dynamics requires the use of numerical models to simulate the phenomenon. Previous numerical models have not incorporated all aspects of the air pressurization in their formulation. In this chapter, we present a study comparing two different model approaches. The first model is based on the assumption that the pressurized portion of the flow may be described by a rigid column approach, and makes use of the method of the characteristics (MOC) to describe the free surface portion of the flow. The second model is based on the Preissmann Slot method, which allows the sewer surcharging, but also permits the application of openchannel equations throughout the pipe. Within this method two different numerical schemes were compared: the Lax-diffusive scheme, and the

Vasconcelos, J. and S.J. Wright. 2004. "Numerical Modeling of the Transition between Free Surface and Pressurized Flow in Storm Sewers." Journal of Water Management Modeling R220-10. doi: 10.14796/JWMM.R220-10.

(C) CHI 2004 www.chijournal.org ISSN: 2292-6062 (Formerly in Innovative Modeling of Urban Water Systems. ISBN: 0-9683681-9-0) 
Lax-Wendroff predictor-corrector scheme. The numerical results were compared with results from a laboratory model, comprising a $14.8 \mathrm{~m}-1 \mathrm{long}, 9.4 \mathrm{~cm}-$ diameter pipeline. In the model, inflow was suddenly initiated into a fill box at one end of the model, while at the other end there was a surge chamber in which the water level was recorded with a digital camera during the pipeline filling process. Measurements of water depth variation with time were obtained at selected pipe sections during the filling process. In particular, the motion induced by the pressurization of the air was recorded to document the effect.

The rigid column model, despite its conceptual simplicity, is able to predict the fill box behavior fairly well, although it is not able to give a detailed pressure distribution along the pipeline, which might be of importance in some applications. On the other hand, the Preissmann Slot based models were able to predict that detailed description, both during the pipe filling process and subsequently, when closed pipe transients are observed. While numerical diffusion is present in the results presented by the Lax-diffusive scheme, the Lax-Wendroff solution was characterized by pressure oscillations at the moving flow regime interface that are considered to be due to numerical rather than physical processes. Future model improvements will be guided by additional experiments as well as implementation of a more sophisticated numerical scheme.

\subsection{Introduction}

Stormwater drainage systems, which are designed to operate in free-surface conditions, may undergo transition to pressurized flow conditions during intense rain events. This flow regime transition is characterized by the propagation of a pressurization front in the form of a non-linear wave, or hydraulic bore. During this process, high-pressure peaks may occur inside sewers, causing structural damages to the system, geysering through manholes or other vertical shafts, and other operational problems, as related by Zhou et al. (2002), and Guo and Song $(1990,1991)$ among others.

The flow regime transition in stormwater sewers occurs whenever the flow depth exceeds the pipe diameter, causing the pressure increase from the initial atmospheric. During conditions of high inflow rates, the transition may develop as a pipe-filling bore. The transient nature of the flow in these conditions can only be described by solving the complete continuity and momentum balance equations. Because the closed sewers are initially partially filled with air, the problem is actually a two-phase transient flow, but most of the studies to date consider only the water phase in the analysis. 
Some stormwater models describe inadequately the flow in sewers during strong rains either by not considering the flow regime transition or by doing this improperly. Some model approaches, not considering the dynamic nature of the filling process, are unable to describe the formation of a pressurization front. For instance, Wright et al. (2003) reported that high hydraulic grade line spikes occurred while running SWMM for the high flows associated with the filling of a below grade storage tunnel with the design rainfall. They interpreted these to be related to the fact that SWMM does not accurately represent bores that could develop in a rapidly filing tunnel. They reasoned that the model code is apparently constrained to fill no more than one model segment in a time step, effectively artificially fixing the bore speed by the model spatial and temporal discretization.

Different approaches to the description of the flow regime transitions have been developed to date. These approaches are divided in two groups: Interface tracking approaches and Preissmann slot approaches. The interface tracking methods are characterized by the monitoring of the pressurization interfaces during the simulation, during which the appropriate set of equations is used in each flow regime domain. Examples of such approaches are the models proposed by Wiggert (1972), Sundquist and Papadakis (1982) and Cardle and Song (1988). The Preissmann slot techniques are characterized by the introduction of a hypothetical narrow slot at the top of the sewers. This feature was first proposed by Cunge and Wegner (Cunge et al., 1980) and allows the application of the Saint-Venant equations throughout the flow domain. Different numerical approaches to the solution of the Saint-Venant equations yield different numerical formulations. Examples of the use of the Preissmann Slot technique are provided by Garcia-Navarro et al. (1994), Capart et al. (1997), Ji (1998) and Trajkovic et al. (1999).

Most of the current approaches for the description of the flow regime transition implicitly assume that the air phase within the system is evacuated without significant restriction. As a consequence, the air phase is not considered in the description of the flow dynamics. Some authors consider the presence of the air phase as a complicating factor in the analysis. Trajkovic et al. (1999) stated that insufficient air ventilation is one of the causes of flow instabilities. According with the authors, those instabilities prevent the application of the Saint Venant because of the two-phase flow occurrence. Even so, air phase may get pressurized in systems with inadequate venting, and in such conditions the effects of the air pressurization to the system dynamics may not be neglected, as indicated by the experimental results presented by Vasconcelos and Wright (2003a). 


\subsection{Review of Flow Regime Transitions Models with Restricted Venting Conditions}

Several approaches to describe the flow regime transition have incorporated some of the effects of the air dynamics in the system. One approach was proposed by Hamam and McCorquodale (1982). Their work accounted for the air pressure ahead of the advancing surcharge bore to describe the transition between the flow regimes. During the flow regime transition, the pressurized portion behavior is modeled through a rigid column approach. Upon full system pressurization, the flow is solved through the method of the characteristics MOC - accounting for the reduction of the wave speed caused by the air present in the water phase. Their approach, though, does not discuss how to obtain the air pressure ahead of the moving bore.

$\mathrm{Li}$ and McCorquodale (1999) improved the Hamam and McCorquodale (1982) approach in the sense that they created a mechanism for bubble creation, movement and release out of the pipe. This mechanism is based on the interface instability that would be expected when the air phase, traveling over the water phase, generates waves in the water that may touch the pipe crown, creating thus the bubbles. They presented a model for the mixed flow in storm sewers in which the pressurized and free surface portions of the flow are treated as rigid columns, and conducted laboratory experiments to calibrate and verify their model. To calculate the air pressure, their model assumes the air undergoes a pseudo-adiabatic process, and uses this in a mass balance equation for the air phase.

Another approach was proposed by Zhou et al. (2002), who studied the problem of the water advance through an initially empty pipe assuming a vertical interface. Also attempting to model stormwater flow transitions using a rigid column approach, they accounted for the interaction between the air phase pressurization and its effect in the water advance. However, the assumption of the vertical interface separating the flow regimes may be improper for nearly horizontal pipelines. In such cases, the flow transitions would be in the form of a moving bore propagating over a partially filled pipe.

All the mentioned approaches have considered the effects of the air phase pressure in the sense that it slows down the advance of the water pressurization front. In each of the models it is considered the air pressure force acting against the interface, which causes a decrease in the bore velocity. Thus, the models interrelate the advance of the water phase and the air pressure, and the proposed methods account for this interrelationship in their analyses. But the effects of 
the air phase in the flow regime transition are not restricted only to the retardation of the bore propagation, as other flow features associated with the air pressurization have been detected.

Experiments reported by Wright et al. (2003) indicate some additional flow features were observed in the sewers subjected to air pressure during the filling process. One feature, referred by the authors as pre-bore motion, was the movement of the water in the free surface region prior to the bore arrival. As indicated in Figure 10.1, the sudden inflow admission when the system was at rest caused a bore that moved towards the riser end. In cases where the water depth increases in the direction of the bore movement, it was possible that the air escape at the riser was restricted sufficiently to cause pressurization of the air phase. As a result, during the pre-bore motion, water flow towards the riser was observed, while the bore was still located close to the fill box, as indicated in Figure 10.1. Eventually, the riser water level rose above the pipe crown, and air bubbling developed at that location until the bore arrived at the riser. This result was initially unexpected because the bore (in the absence of the air pressurization) is the fastest moving nonlinear disturbance, and no fluid motion was expected prior to the bore arrival. Additional experiments were conducted to determine the causal relationship between the air pressure and the pre-bore motion.

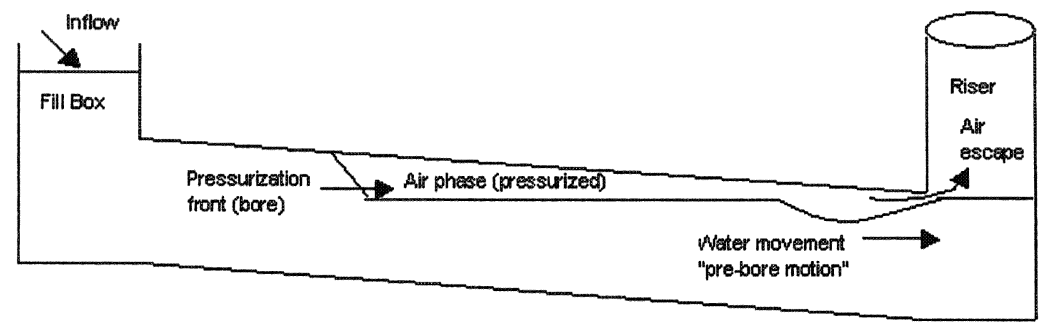

Figure 10.1 Schematics of the bore propagation in a sloped pipe. Vertical scale is exaggerated

To further study this flow feature, Vasconcelos and Wright (2003b) conducted experiments in which the inflow was admitted into a horizontal pipeline, with the venting allowed through a venting tower, as indicated in Figure 10.2. Various sizes of restrictor nozzles could be inserted in the top of the venting tower to restrict the escape of air from the model. In these conditions it was also possible to observe the occurrence of the pre-bore motion, and a numerical model was constructed to simulate the experiments. 
The model was based on the interface tracking approach, solving the pressurized portion of the flow by applying the momentum balance equation in a water column that represented the pressurized region, while the free surface was solved by the MOC. The numerical model was successful in simulating the general features of the observed results.

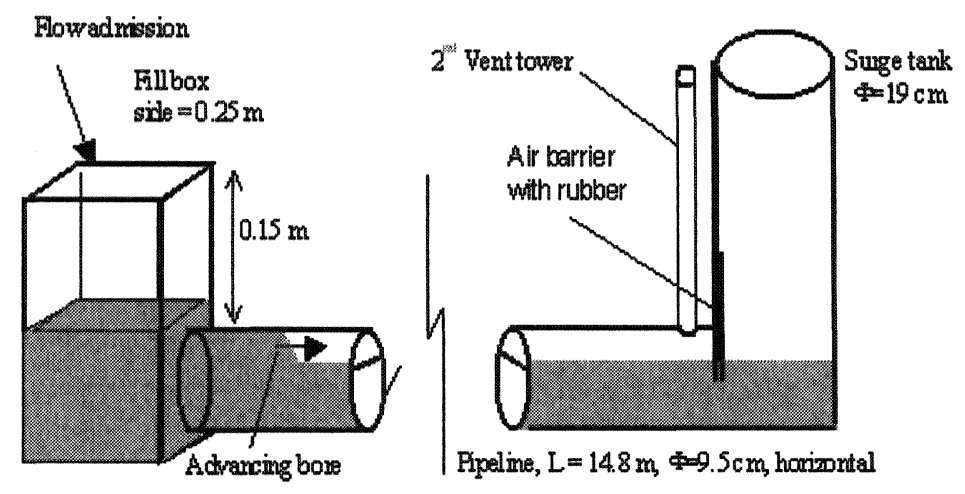

Figure 10.2 Sketch of apparatus used to study the pre-bore motion feature.

The authors also reported a second flow feature not accounted in the current models, which was an air wedge intruding over the pre pressurization bore, referred as air-counter flow. This feature was observed when the smallest venting nozzle was used, and thus it is related to the development of greater air pressures within the pipe. In such conditions, the hydraulic bore movement was partially retarded along the pipeline and an air wedge formed at the top of the bore. The wedge then started to move back towards the fill box, while the hydraulic bore continued its movement towards the riser but without filling the pipe cross section. The air wedge movement occurred in the same manner as a gravitational current with distinct front such as described by Benjamin (1968) and others. Figure 10.3 sketches the general configuration of the feature. The model by Vasconcelos and Wright (2003b) has not tried to include this feature in the formulation.

Though the model was able to provide a reasonable description of the observed results, it suffers from some limitations. The assumption of pressurized flow behind the bore requires an estimation of the initial length of the pressurized column as an initial condition, and this needs to be estimated in some ad-hoc fashion. In addition to this, the rigid column formulation used in the 


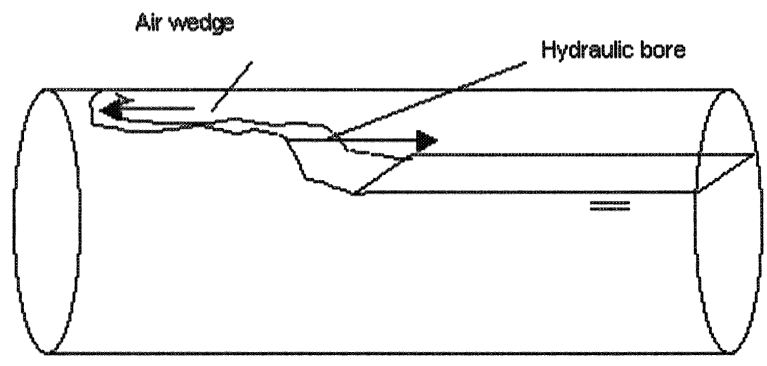

Figure 10.3 Sketch of the resulting configuration when the air wedge appeared over the bore.

model does not allow a variation in flow variables at the internal nodes in the system during this transient phase. Because the internal pressures in the system may exceed the pressure observed in the system boundaries, the additional information may be desirable in an application.

These limitations may be addressed with the use of a discrete numerical approach, which would be able to describe the flow from the initiation moment and provide information on the internal nodes. Different alternatives are available for this purpose, such as Finite Difference or Finite Volume methods, coupled with the Preissmann slot method or the MOC used with a shock-fitting technique as an example of the interface tracking methods.

Summarizing, some stormwater flow models are able to describe the rapid filling pipe process occurring in stormwater systems. The accuracy of such description requires the appropriate treatment for the flow regime transition, which includes the formation and propagation of the pressurization bore that interfaces the different flow regime regions. These models usually assume perfect venting conditions in the systems, so that the air phase does not play a role in the system dynamics. Few approaches to model the flow regime transition have considered the air pressurization to affect the bore propagation. Recent experiments indicated some other flow features that may occur in the water phase beneath the pressurized air phase which were not accounted in existing models. A model was constructed to simulate the pre-bore motion, and it was able to describe the overall behavior of the system. However, it can't describe the initial moments of the flow initiation nor provide information for the internal nodes of the pressurized region, and a discrete numerical model is required for that purpose. 


\subsection{Objectives}

The present work is part of an ongoing research program that aims to incorporate the air pressurization feature in discrete numerical models. The numerical schemes chosen herein are based on the Preissmann Slot technique. The reason for this is the conceptual simplicity of this method, and because the same set of equations are applicable throughout the flow domain, making interface tracking unnecessary. Additionally, the interface-tracking formulation usually requires the solution of a system of non-linear equations, what is cumbersome computationally. The numerical techniques chosen for the present study, the Lax-Diffusive and the Lax-Wendroff schemes, are among the simplest approaches to describe the flow regime transition and the shock formation using the Preissmann slot concept.

The following sections will discuss details of the ongoing development of a discrete numerical scheme able to describe the changes in the bore propagation speed and the pre-bore motion. The alternative discrete approaches to solve the Saint-Venant equations and the concepts of the pressurization model used are discussed. The results from the discrete models are compared with the actual experimental data along with the result from the previous interfacetracking model proposed by Vasconcelos and Wright (2003b). No attempt was made to describe the air counter-flow feature in this study, this being an ongoing research topic.

\subsection{The Saint-Venant equations with the Preissmann Slot}

The Saint-Venant equations, or the continuity and momentum balance equations for the open channel flow, can be written in the conservative, vectorized form:

$$
\begin{aligned}
& \frac{\partial \vec{U}}{\partial t}+\frac{\partial F(\vec{U})}{\partial x}=S(\vec{U}) \\
& \text { in which } \\
& \vec{U}=\left[\begin{array}{l}
A \\
Q
\end{array}\right] \quad F(\vec{U})=\left[\begin{array}{c}
Q \\
\frac{Q^{2}}{A}+g I
\end{array}\right] \quad S(\vec{U})=\left[\begin{array}{c}
0 \\
g A\left(S_{o}-S_{f}\right)
\end{array}\right]
\end{aligned}
$$


where:

$$
\begin{aligned}
A= & \text { cross sectional area of the channel, } \\
Q= & \text { flow rate } \\
g= & \text { gravity, } \\
I= & \text { cross-sectional first moment of inertia, } \\
S_{o}= & \text { pipe slope, and } \\
S_{f}= & \text { friction slope, evaluated here using the Manning } \\
& \text { equation, in which } R h \text { is the hydraulic radius: }
\end{aligned}
$$

$$
S_{f}=\frac{(n Q)^{2}}{A^{2} R h^{4 / 3}}
$$

The Saint-Venant equations are the unsteady, mass and momentum equations applicable for open channel flow only. For an arbitrary channel cross-section, the propagation speed $\mathrm{c}_{\mathrm{w}}$ of an elementary disturbance is given by

$$
c_{w}=V \pm \sqrt{\frac{g A}{T}}
$$

where:

$$
\begin{aligned}
& V=\text { flow velocity, and } \\
& T=\text { top width of the channel. }
\end{aligned}
$$

The flow equations would be applicable to describe the unsteady, pressurized flow that would occur in the sewers prior to the pressurization bore arrival. However, Equation 10.4 is meaningless if $\mathrm{T}$ becomes zero, as occurs when the cross section is full.

The Cunge and Wegner approach was to introduce a hypothetical slot at the top of the pipe as depicted in Figure 10.4. The slot width can be selected so that the much larger pressurized flow wave celerity is attained, and thus the same set of equations can be applied to solve the flow regime transition. The width also should be kept small, so the effect on the continuity equation is minor. The great advantage is that the different flow regime regions are solved with the same set of equations, as opposed to the interface tracking models. On the other hand, one disadvantage of the Preissmann Slot is that it allows the water level to drop below the pipe crown in when the pressure drops, even if there's no venting point at that location. But for the purposes of the present analysis this is not important, since during the experiments, once the system became fully 
pressurized, the system never reverted to a free surface flow regime. In any case, a full implementation of the model will require the air release only in discrete locations where venting is available.

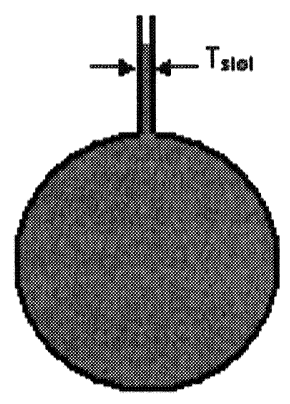

Figure 10.4 Scheme of the Preissmann Slot at the top of a pressurized sewer.

\subsubsection{Lax-Diffusive Approach}

The Lax-Diffusive approach solves the Saint-Venant equations in a discrete domain by forming the time derivative at the neighboringnodes. The discretization of the Saint-Venant equations is represented in the form of Equations 10.5 and 10.6 (Sturm 2001, Katopodes, 2002), with $\mathrm{i}$ as the space index and $\mathrm{k}$ as the time index. As observed in the equations and in Figure 10.5, the calculation of the area and flow rate at any point for the next time step depends only in the neighboring points at the present time step.

$$
\begin{aligned}
& A_{i}^{k+1}=\frac{1}{2}\left(A_{i-1}^{k}+A_{i+1}^{k}\right)-\frac{\Delta t}{2 \Delta x}\left(Q_{i+1}^{k}-Q_{i-1}^{k}\right) \\
& Q_{i}^{k+1}=\frac{1}{2}\left(Q_{i-1}^{k}+Q_{i+1}^{k}\right)-\frac{\Delta t}{2 \Delta x}\left[\left(\frac{Q^{2}}{A}+g I\right)_{i+1}^{k}-\left(\frac{Q^{2}}{A}+g I\right)_{i-1}^{k}\right]+\Delta t\left(\frac{\phi_{i+1}^{k}+\phi_{i-1}^{k}}{2}\right)
\end{aligned}
$$

The presented Lax-Diffusive scheme is an example of explicit numerical schemes, and as is well known, the time steps for the calculations can't be arbitrarily long for explicit schemes. The stability of the numeric model is conditioned to the observation of the Courant-Friedrich-Levy condition, which states the maximum allowed time step in the model: 


$$
\Delta t \leq \frac{\Delta x}{\left|V \pm \sqrt{\frac{g A}{T}}\right|}
$$

where:

$$
T=\text { narrowest free surface width at the given channel }
$$
cross section.

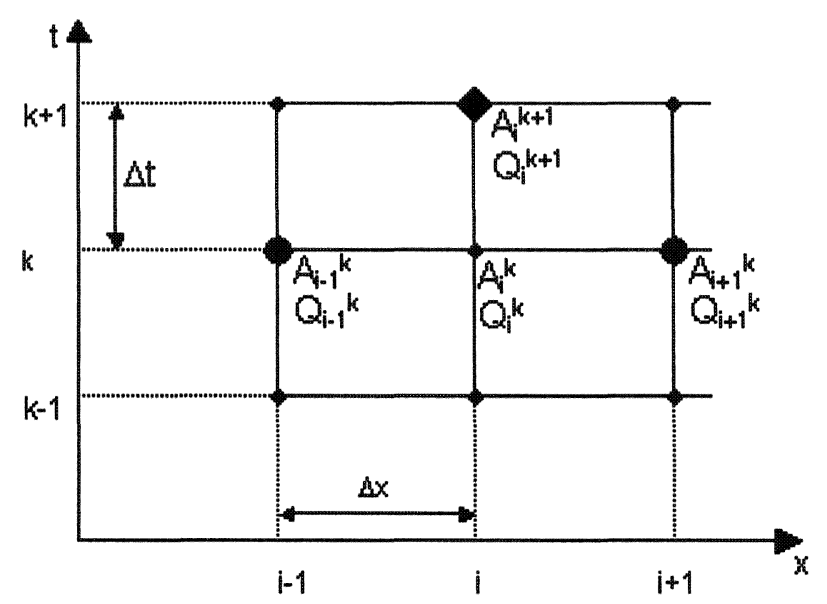

Figure 10.5 Computational nodes for the Lax-Diffusive scheme (adapted from Sturm, 2001). Note that the calculation of the next time step values depend only on the neighboring points. (Bigger dots: source information nodes; diamond: newly calculated nodes).

\subsubsection{Lax-Wendroff Scheme}

The Lax-Wendroff scheme is one example of a shock-capturing, predictorcorrector numerical scheme. It computes the values for area and discharge in each node in a two-step process, the predictor step and the corrector step. For the predictor step, when $T=(k+1 / 2) \Delta t$, the values for $A$ and $Q$ in each node are calculated according to the Lax-Diffusive scheme. For the corrector step, the Leapfrog scheme (Sturm 2001) is used to calculate the final values for A and Q.

Thus, for the first half of the time step (predictor step), the Lax-Wendroff scheme discretizes the Saint-Venant in the following form: 


$$
\begin{aligned}
& A_{i}^{k+1 / 2}=\frac{1}{2}\left(A_{i+1}^{k}+A_{i}^{k}\right)-\frac{\Delta t}{2 \Delta x}\left(Q_{i+1}^{k}-Q_{i}^{k}\right) \\
& Q_{i}^{k+1 / 2}=\frac{1}{2}\left(Q_{i+1}^{k}+Q_{i}^{k}\right)-\frac{\Delta t}{2 \Delta x}\left[\left(\frac{Q^{2}}{A}+g I\right)_{i+1}^{k}-\left(\frac{Q^{2}}{A}+g I\right)_{i}^{k}\right]+\frac{\Delta t}{2}\left(\frac{\phi_{i+1}^{k}+\phi_{i}^{k}}{2}\right)
\end{aligned}
$$

For the second half of the time step (corrector step), the Lax-Wendroff scheme discretizes the Saint-Venant in the following form:

$$
\begin{aligned}
& A_{i}^{k+1}=A_{i}^{k}-\frac{\Delta t}{\Delta x}\left(Q_{i+1 / 2}^{k+1 / 2}-Q_{i-1 / 2}^{k-1 / 2}\right) \\
& Q_{i}^{k+1}=Q_{i}^{k}-\frac{\Delta t}{\Delta x}\left[\left(\frac{Q^{2}}{A}+g I\right)_{i+1 / 2}^{k+1 / 2}-\left(\frac{Q^{2}}{A}+g I\right)_{i-1 / 2}^{k+1 / 2}\right]+\Delta t\left(\frac{\phi_{i+1 / 2}^{k+1 / 2}+\phi_{i-1 / 2}^{k+1 / 2}}{2}\right)
\end{aligned}
$$

The resulting computational grid is indicated in Figure 10.6.

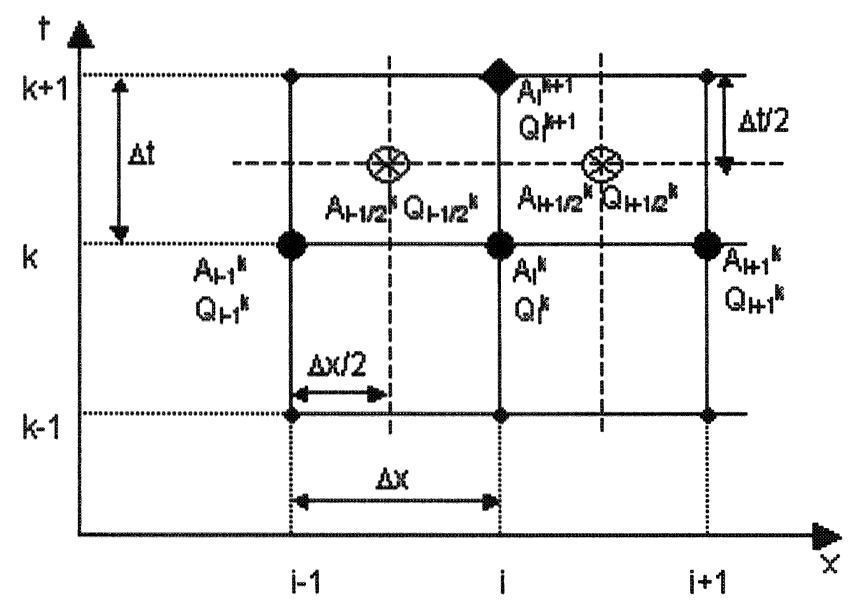

Figure 10.6 Computational nodes for the Lax-Wendroff scheme (adapted from Sturm, 2001). Note that the calculations are conducted in two steps. (Filled dots: source information nodes; white dots: predictor step results; diamond: newly calculated nodes).

One characteristic problem of the Lax-Wendroff approach is the generation of numerical fluctuations at the vicinity of the shock. The problem has been addressed by a number of researchers in the sense that some artificial viscosity was introduced to restrict these oscillations. The oscillations have a more severe 
impact is describing the flow transition using the Preissmann slot, since any small oscillation in the calculated area of a pressurized cross section represent a significant change in the head, which in turn affects the momentum calculation. However, no attempt to include improvements in the original formulation was made in this study, since the focus of this initial work is to assess whether the coupling of the air pressure with a discrete approach is possible.

\subsubsection{Air Pressurization Modeling and Coupling with Water Flow Simulation}

An accurate description of the air phase flow during the rapid filling process is a complex task in the sense it would require a fully transient approach accounting for the changes in the air phase boundaries and geometry along the process. This would be especially necessary in simulations of actual stormwater drainage systems that have large $\mathrm{L}_{\mathrm{s}} / \mathrm{a}_{\text {air }}$ time scales, in which $\mathrm{L}_{\mathrm{s}}$ is the distance between venting points within the system and $a_{\text {air }}$ the wave speed of the air transients. A general solution procedure should model the transient nature of the two-phase flow allowing for different air pressures along the system and for air compressibility..

However, most systems should have sufficient venting to be able to neglect the complete air dynamics as well as the compressibility. A simple mass balance equation for the air phase and a relationship between the discharged air and the air pressure head then are basically the requirements to include the air in the system dynamics in a simplified fashion. The low air pressures expected and the range of the air speed at the experimented conditions are such that we assumed the air phase to be essentially incompressible. It is not difficult to implement the effects of air compressibility in the formulation, but unnecessary for the interpretation of the present experimental results

The relationship between the air phase and discharge, based on Zhou et al. (2002), is the following:

$$
Q_{\text {air }}=C_{d} A_{\text {orif }} \sqrt{2 g \frac{\rho_{\text {water }}}{\rho_{\text {air }}}\left(H^{*}-H_{b}^{*}\right)}
$$

where:

$$
\begin{aligned}
Q_{a i r} & =\text { air discharge, } \\
C_{d} & =\text { air outlet discharge coefficient, } \\
H^{*} \text { and } H_{b}^{*} & =\text { respectively the absolute air pressure and } \\
\rho_{\text {air }} \text { and } \rho_{\text {water }} & =\text { densities of the air and water phases. }
\end{aligned}
$$


By applying continuity of the air phase, basically dependent on the amount of the water that enters and exits the system, and with the use of Equation 10.12 to relate $\mathrm{Q}_{\mathrm{air}}$ and $\mathrm{H}_{\mathrm{air}}$, the following relation was derived for the air pressure head:

where:

$$
H_{\text {air }}=\frac{1}{2 g} \frac{\rho_{\text {air }}}{\rho_{\text {water }}}\left[\frac{Q_{\text {in }}-Q_{\text {out }}}{C_{d} A_{\text {orif }}}\right]^{2}
$$

$$
\begin{aligned}
H_{\text {air }}= & \text { air phase pressure head, and } \\
Q_{\text {in }} \text { and } Q_{\text {out }}= & \text { amount of water entering and exiting the tunnel in a } \\
& \text { given time step, respectively. }
\end{aligned}
$$

The resulting value for the $\mathrm{H}_{\text {air }}$ is an input parameter both in the pressurized and free-surface portions of the flow and it represents the coupling between the air phase model and the water phase simulation. The change to the solution of the flow regime transition is essentially made by incorporating the pressure force caused by the air phase in the momentum equation. It is assumed that in the nodes in which the flow depth is below the pipe crown, the value for the air pressure head is the one calculated in Equation 10.13, being zero if the node is in a cross section totally filled with water. The assumption is that the air pressure head is distributed uniformly on the pipe cross-section, and the pressure force is applied at the pipe centroid.

The resulting format for the Saint-Venant equations, which should be reflected in each the numerical scheme discretization, are represented by the following set of equations:

$$
\frac{\partial \vec{U}}{\partial t}+\frac{\partial F(\vec{U})}{\partial x}=S(\vec{U})
$$

in which, for locations without air phase

$$
\vec{U}=\left[\begin{array}{l}
A \\
Q
\end{array}\right] \quad F(\vec{U})=\left[\begin{array}{c}
Q \\
\frac{Q^{2}}{A}+g I
\end{array}\right] \quad S(\vec{U})=\left[\begin{array}{c}
0 \\
g A\left(S_{o}-S_{f}\right)
\end{array}\right]
$$

and for locations with air phase

$$
\vec{U}=\left[\begin{array}{l}
A \\
Q
\end{array}\right] \quad F(\vec{U})=\left[\begin{array}{c}
Q \\
\frac{Q^{2}}{A}+g I+g A_{\text {pipe }} H_{\text {air }}
\end{array}\right] \quad S(\vec{U})=\left[\begin{array}{c}
0 \\
g A\left(S_{o}-S_{f}\right)
\end{array}\right]
$$

where:

$$
A_{\text {pipe }}=\text { cross sectional area of the pipe. }
$$




\subsubsection{Boundary Conditions}

This section describes how the boundary conditions are described during the pressurization process. The system boundaries are treated using a finite difference scheme to describe the water level in the tanks, coupled with appropriate characteristic and conservation equations. The unknowns at the upstream boundary, at the fill box, are the inflow into the pipe and the change in the depth at the fill box and the first node. To solve for these three equations, we used the continuity equation, the energy equation between the fill box and the pipe beginning, and the $\mathrm{C}^{-}$characteristic equation. The variables $\mathrm{S}_{\mathrm{fS}}, \mathrm{H}_{\mathrm{S}}, \mathrm{V}_{\mathrm{S}}$ and $\mathrm{C}_{\mathrm{S}}$, from the characteristic equations, are respectively the averaged values for the energy slope, depth, velocity, and celerity, calculated according to the Hartree MOC method, as described by Sturm (2001).

$$
\begin{aligned}
H_{f b}^{t} & =H_{f b}^{t-1}+\Delta t\left(\frac{Q_{i}}{A_{f b}}-\frac{A_{1}}{A_{f b}} V_{1}-Q_{\text {overflow }}\right) \\
V_{1} & =V_{S}-\frac{g H_{1}}{c_{S}}-\frac{g H_{S}}{c_{S}}-g\left(S_{f S}-S_{o}\right) \Delta t \\
H_{1} & =H_{f b}^{t}-V_{1} \frac{\left|V_{1}\right|}{2 g}\left[1+K_{f b}\right]
\end{aligned}
$$

where:

$$
\begin{aligned}
& H_{f b}{ }^{t}=\text { fill box water level at given time step, } \\
& \Delta t=\text { time step, } \\
& Q_{i}=\text { inflow rate, } \\
& Q_{\text {overflow }}= \text { overflowed water if the fill box is full (function of } \\
& \mathrm{H}_{\mathrm{f}}^{\mathrm{t}-1} \text { ) } \\
& A_{f b}= \text { cross-sectional area of the fill box } \\
& A_{1} \text { and } V_{1}=\text { cross-sectional area of the pipe and velocity atnode } 1 \\
& S_{o}=\text { channel slope, } \\
& K_{f b}=\text { localized loss coefficient at the fill box exit. }
\end{aligned}
$$

A similar approach is used to calculate the downstream boundary condition, at the riser tank. We need to satisfy continuity, energy balance between the last node of the pipe and the riser, and the characteristic equations. Similarly to the upper boundary condition, the values of $S_{f R}, H_{R}, V_{R}$, and $C_{R}$, are averaged values from the $\mathrm{C}^{+}$characteristic equation, respectively the energy 
slope, depth, velocity, and celerity, calculated according to the Hartree MOC method (Sturm, 2001).

$$
\begin{gathered}
H_{\text {riser }}^{t}=H_{\text {riser }}^{t-1}+\Delta t \frac{A_{N O}}{A_{\text {riser }}} V_{N O} \\
H_{N O}=V_{N O} \frac{\left|V_{N O}\right|}{g}\left[\left(\frac{A_{N O}}{A_{\text {riser }}}\right)^{2}+\frac{K_{\text {riser }}}{2}\right]+H_{\text {riser }}-H_{\text {air }} \\
V_{N O}=V_{R}+\frac{g H_{R}}{c_{R}}-\frac{g H_{N O}}{c_{R}}-g\left(S_{f R}-S_{o}\right) \Delta t
\end{gathered}
$$

where:

$$
\begin{aligned}
H_{r i s e r}^{t} & =\text { riser water level at given time step, } \\
\Delta t & =\text { time step, } \\
A_{\text {riser }} & =\text { cross-sectional area of the riser, } \\
A_{N O} \text { and } V_{N O} & =\text { cross-sectional area of the pipe and flow velocity at } \\
& \text { the last node, } \\
S_{o} & =\text { channel slope } \\
H_{\text {air }} & =\text { air pressure head, and } \\
K_{\text {riser }} & =\text { localized loss coefficient at the riser entrance. }
\end{aligned}
$$

Based on initial data or on the previous time step results, the boundary conditions are calculated. Then, the code determines whether the inflow causes the pressurization bore that fill the pipe cross-section, triggering the air phase pressurization. In this case, the air phase pressure is calculated through Equation 10.13, and the Saint-Venant equations are solved numerically at each computational node either with Equations 10.2 or 10.14. Figure 10.7 presents the structure of the proposed numerical scheme.

\subsection{Model Results}

Several comparisons between experimental and simulated data were performed to assess the performance of the proposed models. The experimental data compared here was collected by Vasconcelos and Wright (2003a). It included depth measurements in some cross-sections along the pipeline during the rapid filling pipe process and oscillations observed in the surge tank, sketched in 


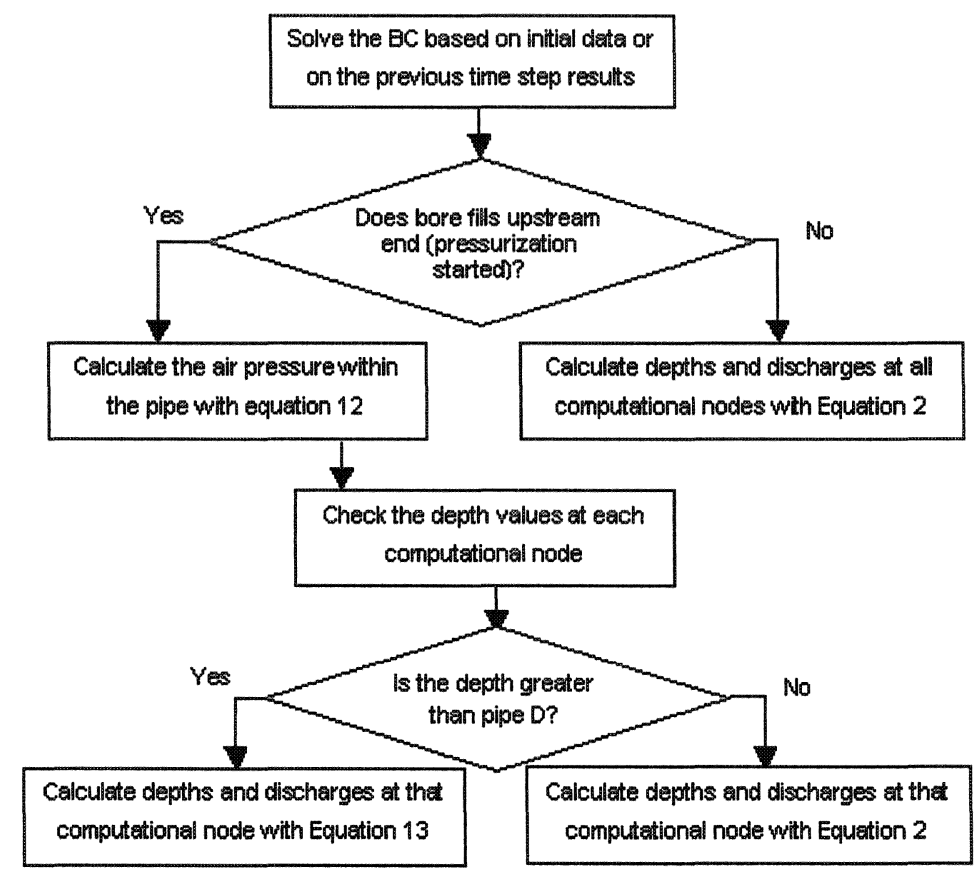

Figure 10.7 Diagram of the model calculation structure within a time step.

Figure 10.2. In addition to this, the experimental results are compared with the predictions of the model presented by Vasconcelos and Wright (2003b).

This study selected some of the collected data and the respective results from the model presented by Vasconcelos and Wright (2003b), hereafter referred to as the rigid column approach. The experimental conditions presented are for a flow rate of $4.1 \mathrm{~L} / \mathrm{s}$ and two initial water depths $(6.5 \mathrm{~cm}$ and $7.1 \mathrm{~cm}$ ). In these experimental conditions, the measured and modeled behavior of the depth at the section $13.9 \mathrm{~m}$ from the fill box end and of the water level at the riser tank were compared. For the purposes of this study, the chosen slot width was $0.0001 \mathrm{~m}$, which yielded a $2 \mathrm{~L} / \mathrm{a}$ time scale around 1 second.

\subsubsection{Depth Behavior at the $13.9 \mathrm{~m}$ Section}

The measurements at the $13.9 \mathrm{~m}$ station show the effect of the air pressurization above the free-surface portion of the flow, specifically water motion prior to the bore arrival. As Figure 10.8 illustrates, as the flow is initiated, a sudden drop 


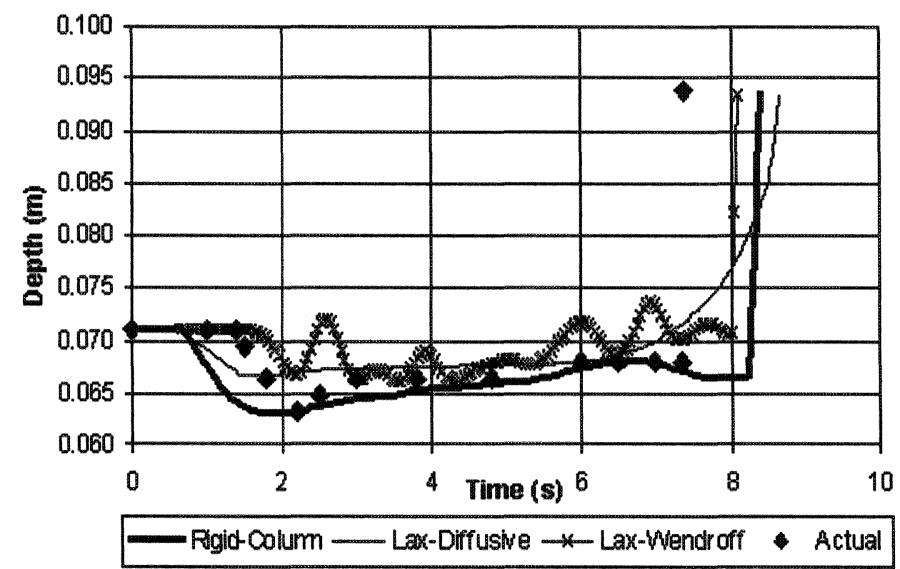

Figure 10.8 Simulation of the water depth behavior at the $13.9 \mathrm{~m}$ section water level $7.1 \mathrm{~cm}$. Note the models generally yield a good description of the depth behavior.

in the section's depth occurs around $1.5 \mathrm{~s}$ from the flow initiation. This drop lasts for a brief moment, and is followed by a slow recovery of the depth at that section. When the bore arrives, at a time of approximately $7.5 \mathrm{~s}$, the depth suddenly jumps to the pipe diameter, signaling the onset of the pressurization at that location.

The different numerical approaches tested here were able to capture the general behavior of the depths as the flow was initiated. Comparing the measurements for the initial depth of $7.1 \mathrm{~cm}$ with the simulation predicted by the Lax-Diffusive approach, one can notice the model's ability to predict the drop in the depth caused by the air pressurization. But the inherent diffusivity of this approach causes problems in the overall timing of the predictions. The moment of the sudden drop occurs earlier, while the predicted bore arrival occurs much later, with a smooth increase of the depth, which doesn't occur in reality. The magnitude of the predicted drop in the depth is reasonably accurate, and smaller than the overpredicted values yielded by the rigid column approach. The timing of the phenomena predicted by these two alternatives is very much comparable.

The comparison between the Lax-Wendroff model and the measured data for the water depth of $7.1 \mathrm{~cm}$ illustrates much better behavior in terms of time response, but also demonstrates the problems caused by the numerical oscillations with this approach. Figure 10.8 shows that the depth drop and the 
bore arrival times are better predicted. However, the numerical oscillations predicted by this model approach prevent us from making an objective assessment of the accuracy of the depth change prediction. Yet, it may be noted that, disregarding the oscillations, the general trend of the depth behavior is reasonably predicted.

The behavior of the different model approaches is generally repeated when the simulation is performed with the initial water level of $6.5 \mathrm{~cm}$, as Figure 10.9 shows. The Lax-Diffusive scheme predicts reasonably well the decrease in the water level, but the timing of the events is again imprecise, with the predicted bore arrival almost $1.5 \mathrm{~s}$ later than the actual arrival time. The Lax-Wendroff approach seems to have a time off-set in the predictions, since both the initial drop in the water depth and the bore arrival occurs about $0.7 \mathrm{~s}$ later than the actual events. However, a decrease in the magnitude of the numerical oscillations can be noticed, and thus a better description of the depth behavior if compared with the results of the higher initial water level simulations.

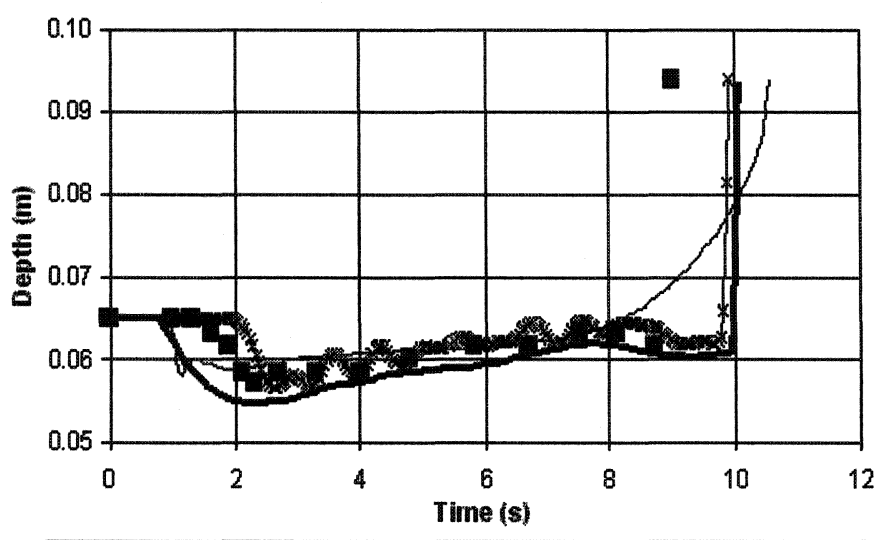

Rigid-Colum $\rightarrow$ - Lax-Wendroff - Lax-Diffusive Experiment

Figure 10.9 Simulation of the water depth behavior at the $13.9 \mathrm{~m}$ section, water level $6.5 \mathrm{~cm}$. The general trend of the simulations is repeated.

The simulations also demonstrated the effect of the Preissman slot opening width in the results, as expected it was found that wider slots causes the bore to arrive later than expected. Capart et al. (1997) suggest that when the flow is not full throughout the pipe the slot width could be set to $10 \%$ of diameter with good results. The reasoning behind this is the much faster celerity this slot width would yield, and thus the transient time scale, compared with the global 
evolution of the filling process. On the other hand a greater slot width will more profoundly affect the continuity equation, since the slot may cause a significant contribution to the cross sectional area.

Additional simulations showed the effect of the Preissmann slot width in the system timing, as the bore arrived later at the riser when wider slots were simulated. Figures 10.10 and 10.11 present the results of the simulations using the Lax-Diffusive approach and the Lax-Wendroff approach respectively with slots widths $10^{-2} \mathrm{~m}, 10^{-3} \mathrm{~m}$ and $10^{-4} \mathrm{~m}$. As the slot width increased, so did the time for the bore to arrive at the $13.9 \mathrm{~m}$ section. That was also observed when the simulations were performed without air pressurization. It was still unclear what causes the difference in the arrival time observed between the simulations with the $10^{-3} \mathrm{~m}$ and $10^{-4} \mathrm{~m}$ slots. In either case, the amount of water stored in the slot is not significant, and continuity alone would not be enough to explain the difference in the arrival time between them.

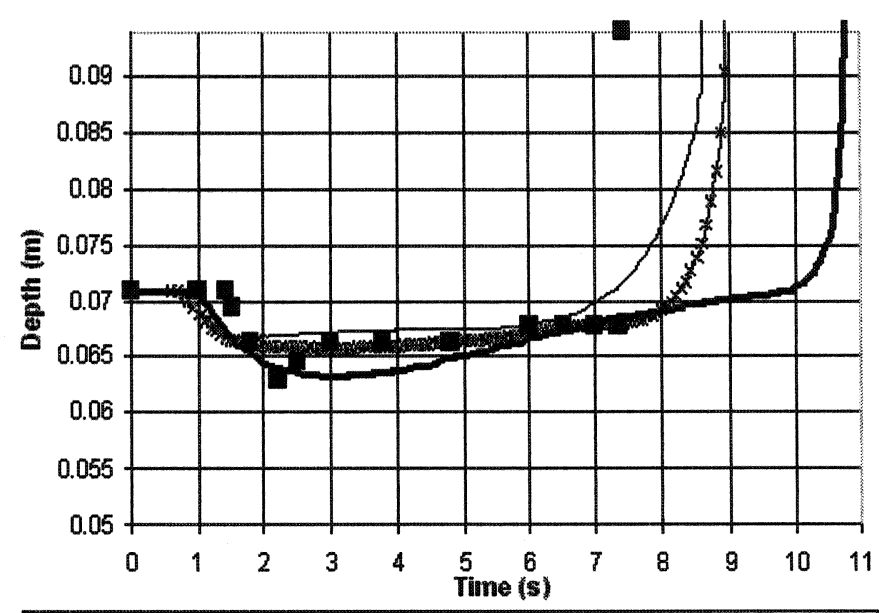

-Slot=1/100 $\mathrm{m} \rightarrow$ Slot $=1 / 1000 \mathrm{~m}-$ Slot $=1 / 10000 \mathrm{~m}$ Actual data

Figure 10.10 Result of Lax-Diffusive simulations for water depth at the 13.9 $\mathrm{m}$ station with different slot widths and initial water level of $7.1 \mathrm{~cm}$. Note the later bore arrival for wider slots.

A better understanding of the relation between the system timing and the slot width could help to improve the description of the bore propagation in the proposed models. The use of the $10^{-4} \mathrm{~m}$ slot in a $94 \mathrm{~mm}$ pipe yields the celerity of approximately $26 \mathrm{~m} / \mathrm{s}$. It is however very hard to know exactly what would be the expected celerity in the pressurized region of the flow. Some values found 


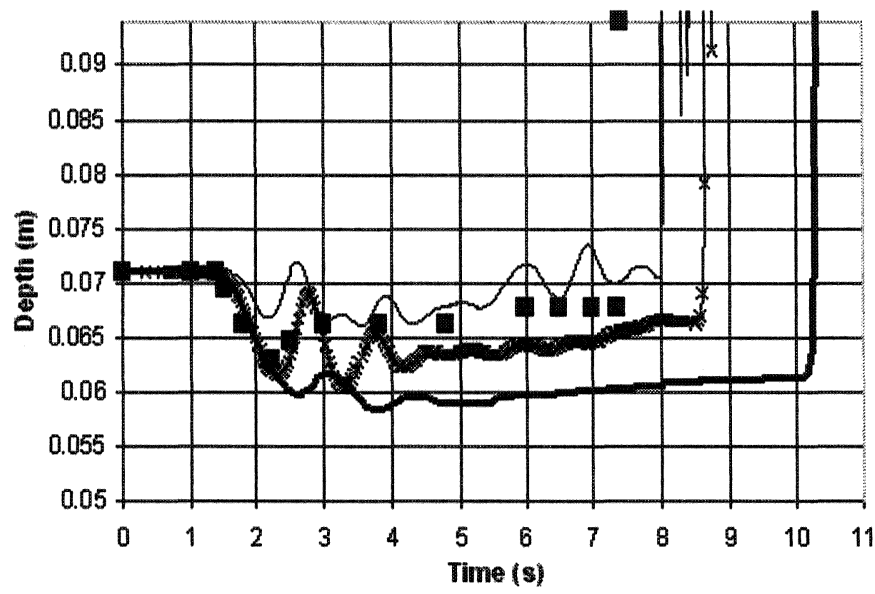

$-S|0 t=1 / 100 \mathrm{~m} \rightarrow-S| 0 t=1 / 1000 \mathrm{~m}-S \mid 0 t=1 / 10000 \mathrm{~m}$. Actual data

Figure 10.11 Result of Lax-Wendroff simulations for water depth at the 13.9 $\mathrm{m}$ station with different slot widths and initial water level of $7.1 \mathrm{~cm}$. Note the later bore arrival for wider slots.

in literature for celerity in a two-phase air/water flow range from $22 \mathrm{~m} / \mathrm{s}$ up to $1440 \mathrm{~m} / \mathrm{s}$ (Hamam and McCorquodale, 1982). Wylie and Streeter suggest that water flowing with $1 \%$ of air would have a wave celerity slightly below $250 \mathrm{~m} / \mathrm{s}$. We believe that if the model has an actual celerity significantly greater than $26 \mathrm{~m} / \mathrm{s}$ it would require a narrower slot in the simulation. This in turn may also help to improve the observed results in terms of timing by speeding up the bore propagation. But this hypothesis requires more investigation since some other factor may be causing the delayed bore arrival predicted in the models.

\subsubsection{Simulation of Riser Tank Motion}

The riser tank predictions showed the ability of the models to describe the general behavior of the air pressurization effects on the surges at the riser tank. The variation of the water level at the riser would be an almost instantaneous increase in the riser water level as a result of the pre-bore motion. Upon the bore arrival at that tank, a steeper increase in the riser water level is then expected. Following the bore arrival, periodical oscillations of the riser water level are expected, with the flow gradually approaching a steady state with all inflow spilling over the top of the fill box. 
As Figure 10.12 illustrates for the initial water level of $7.1 \mathrm{~cm}$, the LaxWendroff scheme provides a very good prediction of the arrival time of the bore at the riser. However, the water level build-up prior to the bore arrival is overpredicted, and that might indicate a problem with the manner in which the air pressure is incorporated in the boundary condition. Fairly good precision in describing the surge peak time and intensity can be noticed. However, the decrease of the water level is overpredicted, and that may be a result of the value of the considered celerity, which may be smaller than the actual celerity. The time offset in the predictions using the rigid column approach to describe the riser water level can also be noticed.

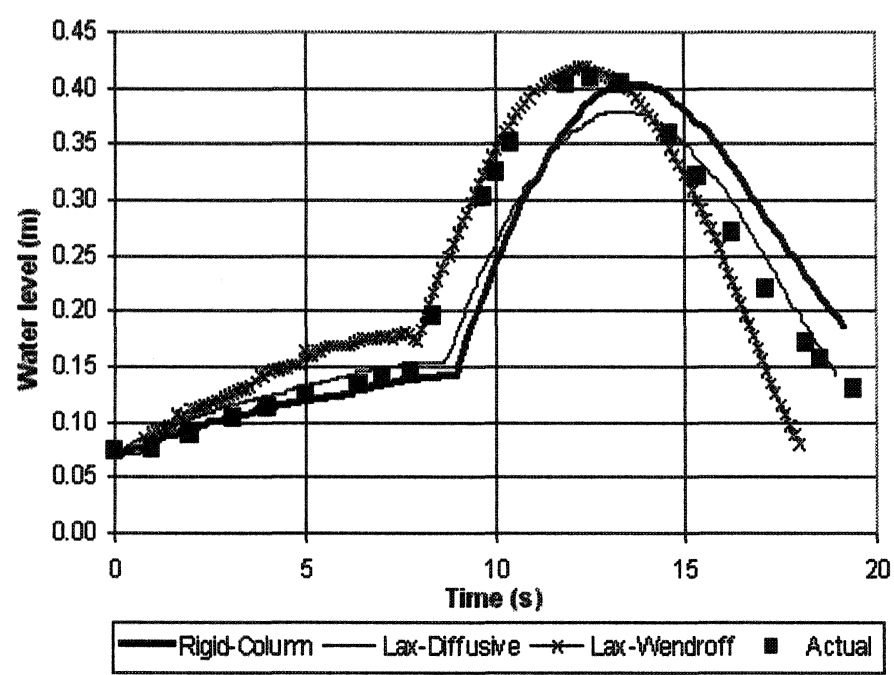

Figure 10.12 Model results for the riser water level in the different approaches. Note the good description for the peak surge using the Lax-Wendroff scheme, while the Lax-Diffusive describes reasonably well the pressure buildup prior to the bore arrival.

On the other hand, the Lax-Diffusive scheme was able to predict very well the pressure build-up observed in the riser prior to the bore arrival. The predicted bore arrival time is later than actual, similar to the description provided by the rigid column approach. Following this, the peak magnitude is underpredicted, and this may be result of the boundary condition. The description of the water level decrease is very good. 
The general pattern is repeated when the initial water level is set to $6.4 \mathrm{~cm}$ in the riser simulations, with the different model approaches providing a better description of the riser behavior compared to the greater initial water level. The Lax-Wendroff scheme again overpredicts the pressure build-up prior to the bore arrival, but this time the difference is not so significant. Yet, there are still problems with the description of the water level decrease. As for the LaxDiffusive approach, the prediction of the data during the pressure build-up phase is again very good, however the bore arrival is later than actual, and the pressure peak is again underestimated. In general, comparing the results of the three different approaches for that figure, the Lax-Wendroff yielded the best description.

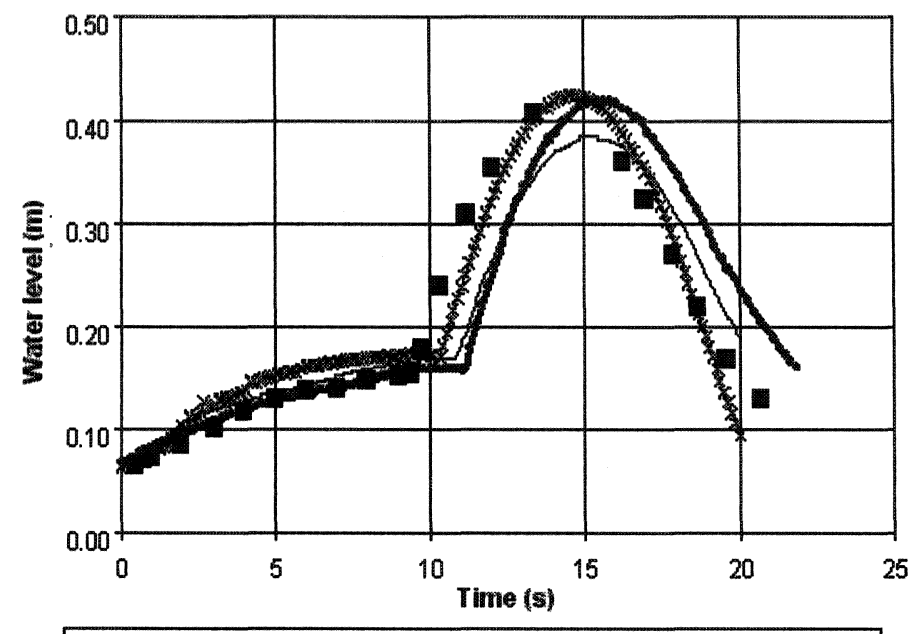

Figio-Colum - Lax-Diffusive $\rightarrow$ - Lax-Wendroff Experiment

Figure 10.13 Model results for the riser water level in the different approaches. Note that the results follow the pattern of Figure 10.12, but the LaxWendroff now has a better overall prediction of the riser water level.

\subsection{Conclusion and Recommendations}

The flow regime transition in storm sewers, which may be expected during intense rain events, can be better described if the air phase is considered in the system dynamics if there are restrictions to the escape of air from the system; a slightly sloping pipeline is apparently sufficient to cause this. The advance of 
the hydraulic bore forces the air phase out of the system, and this result in the pressurization of the air phase, which can be significant especially in poorly ventilated systems. Some model approaches have considered the effects of the air phase in retarding the bore advance, but the effects of the air phase are not restricted to that. Experiments demonstrated the existence of features such as the pre-bore motion and the air counter-flow, which makes the system description even more complex. A numerical model for the flow regime transition was constructed, based on a rigid column approach considering the pre-bore motion feature. But this model has some limitations, which could only be addressed by a discrete numerical scheme.

As a part of an ongoing research, this work studied the feasibility of including air pressurization in the Lax-Diffusive and the Lax-Wendroff schemes to simulate the rapid filling pipe of a storm sewer. Using the concept of the Preissmann Slot, the air phase pressure force was incorporated in the momentum equations. The air phase was described using a simplified approach, based on the air continuity and an orifice equation to relate flow and pressure at discrete venting locations. This approach could simulate the pre-bore motion, but not the yet the air counter-flow feature. The results of the proposed models were then compared with experimental data and with the results of rigid column model previously developed by Vasconcelos and Wright (2002).

The simulation results are in reasonable accordance with the observed data for the water depth at the $13.9 \mathrm{~m}$ section and for the riser water level. It could be noticed the effect of the numerical oscillations in the Lax-Wendroff scheme predictions, but the overall timing of the events was accurate, with a good prediction of the surge peak at the riser. On the other hand, the Lax-Diffusive simulation for the riser tank underestimated the surge peak at the riser, but the pressure build-up resulting from the pre-bore motion was well predicted. The timing predictions of the Lax-Diffusive scheme are not so accurate, and can be compared in this regard with the predictions by the rigid column model. Additionally it was demonstrated the effect of the Preissmann slot width (and thus the simulated wave celerity) in the model results, and it appears that a better understanding of this issue will help improve the model predictions.

Although the various models predict the essential features of the observed flow behavior, it is concluded that there may be problems with each specific numerical method. The future developments points to a numerical model that can give a reasonable treatment of the flow discontinuity, as the Lax-Wendroff scheme, but without numerical instabilities, as the Lax-Diffusive. One alternative is the implementation of the Preissmann Slot using the Finite Volume Method, incorporating the effect of the air phase in the system dynamics. 
Anther future development is related to the air counter-flow feature, basically the conditions when it occurs and how to predict it. Finally, due to the limitations of the Preissmann Slot regarding air release and admission through the slot, a full implementation of the model will require these air transfers only in discrete locations where venting is available. It appears that this can be achieved with a model incorporating features both from the Preissmann Slot and from the interface tracking approaches.

\section{Acknowledgments}

The authors would like to acknowledge the support of the CNPq, a Brazilian Government entity committed to the development of science and technology, which has provided a fellowship to support the $\mathrm{PhD}$. studies of the first author.

\section{References}

Benjamin, T.B. (1968). "Gravity Currents and Related Phenomena," Journal of Fluid Mechanics, 31 (2), 209-248.

Cardle J. A. and Song, C. S. S. (1988). "Mathematical modeling of unsteady flow in storm sewers". International Journal of Engineering Fluid Mechanics, 1 (4), 495518.

Cardle, J. A., Song, C. C. S.; Yuan, M. (1989). "Measurements of mixed transient flows". Journal of Hydraulic Engineering, 115 (2), 169-182.

Capart, H., Sillen X. and Zech, Y.(1997). "Numerical and experimental water transients in sewer pipes". Journal of Hydraulic Research, 35 (5), 659-670.

Chen Y. H. and Yevjevich V. (1977). "Mathematical model of hydraulic transient phenomena in storage and drainage tunnels". Proc. Int. Symp. on Urban Hydrology, Hydraulics and Sediment Control, Lexington, KY, 125-128.

Cunge, J. A., Holly Jr., F. M. and Verwey A. (1980). Practical Aspects of Computational River Hydraulics. Pitman Publishing Ltd., London.

Garcia-Navarro, P., Priestley A., Alcrudo, F. (1994). "Implicit method for water flow modeling in channels and pipes" Journal of Hydraulic Research, 32 (5), 721-742.

Guo Q. and Song, C. S. S. (1990). "Surging in Urban Storm Drainage Systems". Journal of Hydraulic Engineering, $116(12), 1523-1537$.

Guo Q. and Song, C. S. S. (1991). "Dropshaft Hydrodynamics under Transient Conditions". Journal of Hydraulic Engineering, 117 (8), 1042-1055.

Hamam M.A. and McCorquodale J. A. (1982). "Transient conditions in the transition from gravity to surcharged sewer flow". Canadian Journal of Civil Engineering, 9,189-196. 
Ji, Z. (1998). "General hydrodynamic model for sewer/channel network systems". Journal of Hydraulic Engineering, 124 (3), 307-315.

Katopodes, N. (2002). Book Manuscript on Free Surface Flow - Unpublished.

Li J. and McCorquodale A. (1999). "Modeling mixed flow in storm sewers". Journal of Hydraulic Engineering, 125 (11), 1170-1180.

Song, C. S. S., Cardle J. A. and Leung, K. S. (1983). "Transient mixed-flow models for storm sewers". Journal of Hydraulic Engineering, 109 (11), 1487-1504.

Sturm, T. W. (2001). Open Channel Hydraulics, McGraw Hill, New York, 295-305.

Sundquist M. J., and Papadakis C. N. (1982). "Surging in combined free-surface pressurized systems", Proc. ASCE National Convention, Las Vegas, NV, 1-14.

Trajkovic, B., Ivetic, M., Calomino, F., D'Ippolito, A., (1999). "Investigation of transition from free surface to pressurized flow in a circular pipe", Water Science and Technology, 39 (9), 105-112.

Vasconcelos, J.G. and Wright, S.J. (2003a), "Laboratory Investigation of Surges Formed During Rapid Filling of Stormwater Storage Tunnels," Proceedings of 30th IAHR Congress, Thessaloniki, Greece.

Vasconcelos, J.G. and Wright, S.J. (2003b), "Surges Associated with Air Expulsion in Near-Horizontal Pipelines," Proceedings 4th Joint ASME-JSME Fluids Engineering Conference, Honolulu, Hawaii.

Wiggert, D. C. (1972). "Transient flow in free-surface, pressurized systems". Journal of the Hydraulic Division, 98 (HY1), 11-27.

Wright, S.J., J. Vasconcelos and K. Ridgway. 2003. "Surges Associated with Filling of Stormwater Storage Tunnels." Journal of Water Management Modeling R215-18. doi: 10.14796/JWMM.R215-18.

Wylie E. B. and Streeter V.L. (1993). Fluid Transients in Systems. Prentice Hall, Upper Saddle River, NJ.

Zhou, F., Hicks, F. E., Steffler, P. M. (2002). "Transient flow in a rapidly filling horizontal pipe containing trapped air". Journal of Hydraulic Engineering, 128 (6), 625-634. 